\title{
Synthesis of $N$-Fmoc-(2S,3S,4R)-3,4-
}

\section{dimethylglutamine. An Application of Lanthanide-}

\section{Catalyzed Transamidation}

\author{
Selçuk Çalimsiz and Mark A. Lipton* \\ Department of Chemistry and Cancer Center, Purdue University, 560 Oval Drive West Lafayette, IN \\ 47907-2084, USA \\ lipton@purdue.edu
}

Table of Contents

List of Figures: $\quad$ Page S1

Experimental Procedures and Characterizations: Page S2-S6

NMR spectra:

Page S7-S24

\section{List of Figures}

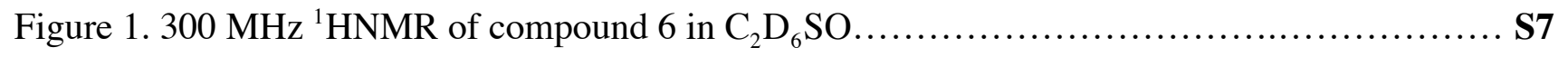

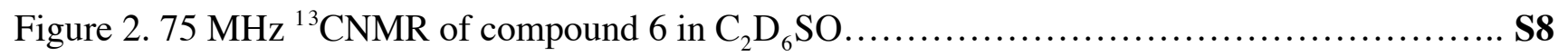

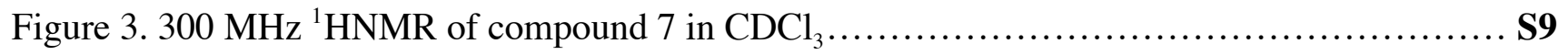

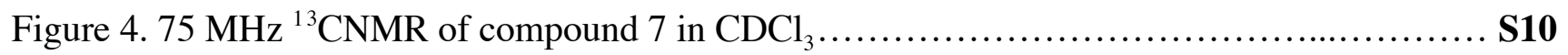

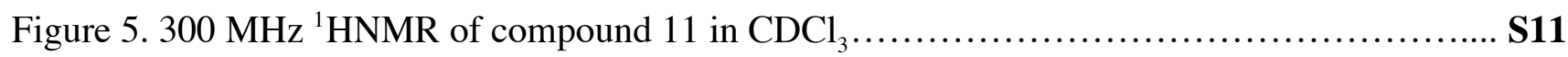

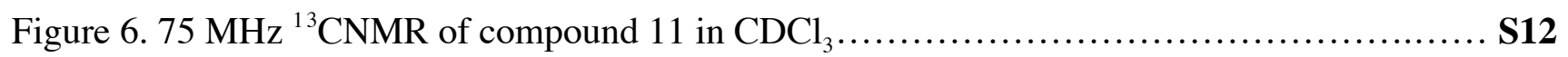

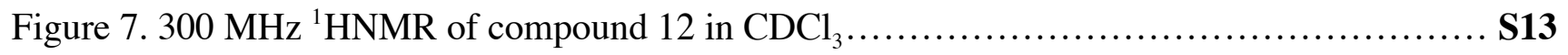

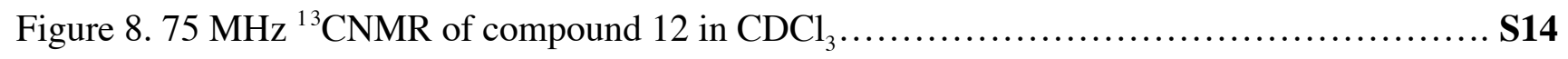

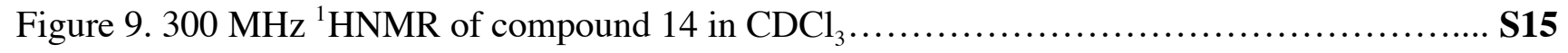




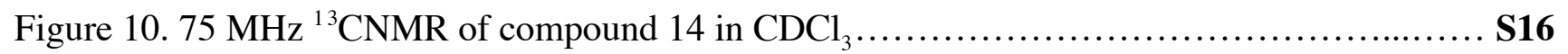

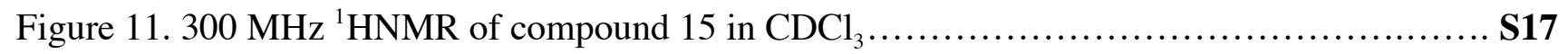

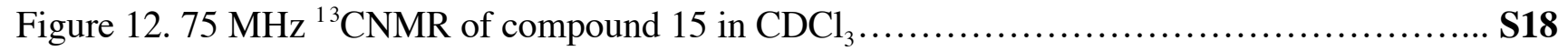

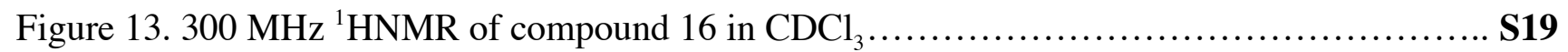

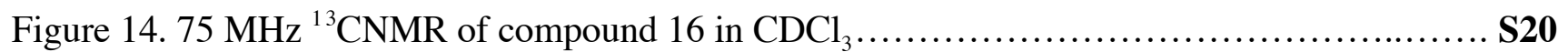

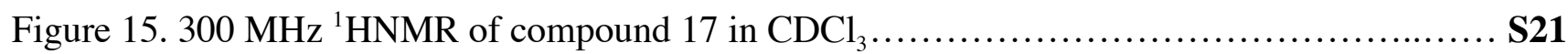

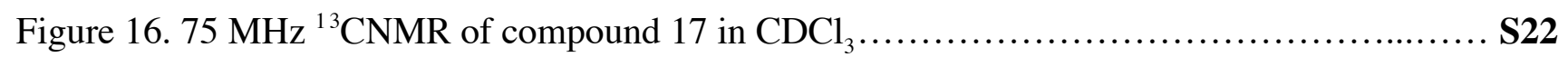

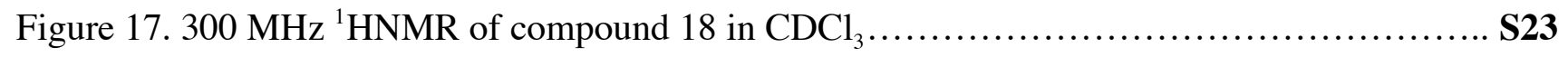

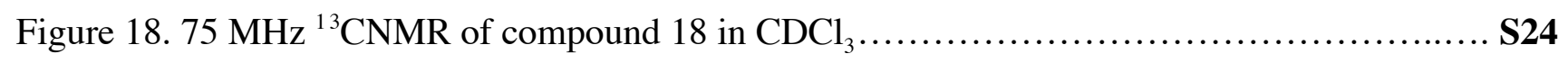

\section{General Experimental}

NMR spectra were measured using at $300 \mathrm{MHz}$ and $75 \mathrm{MHz}$ for proton and carbon nuclei respectively.

IR spectra were recorded in $\mathrm{NaCl}$ pellets. All melting points are uncorrected. All syntheses were carried out under positive nitrogen pressure, and solvents were purified by passage through a solvent column prior to use. ${ }^{1}$ Flash chromatography was carried out using 230 400 mesh silica gel. Compounds $\mathbf{8}^{2}, \mathbf{9}^{3}, \mathbf{1 0}^{4}$ were prepared according to literature procedures.

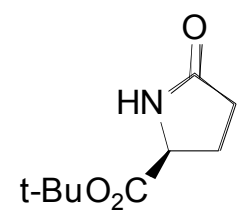

$t$-butyl (R)-pyroglutamate (10). The general procedure for Boc deprotection of lactam was followed using $t$-butyl $N$-Boc-(4R)-pyroglutamate (9) $(33 \mathrm{mg}, 0.116 \mathrm{mmol})$ and $\mathrm{Yb}(\mathrm{OTf})_{3}(32.4 \mathrm{mg}, 0.052 \mathrm{mmol})$. Flash column chromatography with EtOAc yielded $\mathbf{1 0}$ as a colorless, crystalline solid (21.4 mg, $100 \%)$ : mp 104-106 ${ }^{\circ}$; ${ }^{1} \mathrm{H}$ NMR (300 MHz, CD $\left.\mathrm{OD}\right) \delta 4.16$ (dd, 1H, 8.8, $\left.4.5 \mathrm{H}\right), 2.39-2.52$ (m, 1H), 2.36-2.30

\footnotetext{
${ }^{1}$ The solvent columns are composed of activated alumina (A-2) and supported copper redox catalyst (Q5 reactant). See: Pangabom, A. B.; Giardello, M. A.; Grubbs, R. H.; Rosen R. K.; Timmerks, F. J. Organometallics 1996, 15, 1518-1520.

${ }^{2}$ Acevedo, C. M.; Kogut, E. F.; Lipton, M. A. Tetrahedron 2001, 57, 6353-6359.

${ }^{3}$ Kolasa, T.; Miller, M. J. J. Org. Chem. 1990, 55, 1711-1721; August, R. A.; Khan, J. A.; Moody, C. M.; Young, D. W. J. Chem. Soc. Perkin Trans. 1 1996, 507-514.

${ }^{4}$ Siro, J. G.; Martín, J.; Garc_a-Nav_o, J. L.; Remui_an, M. J.; Vaquerro, J. V.; Synlett 1998, 147-148.
} 
(m, 2H), 2.16-2.07 (m, 1H), 1.49 (s, 9H) ppm. ${ }^{13} \mathrm{C}$ NMR (75 MHz, CD $\left.\mathrm{OD}\right) \delta$ 181.3, 173.5, 83.2, 57.9, $30.5,28.3,26.1 \mathrm{ppm}$.

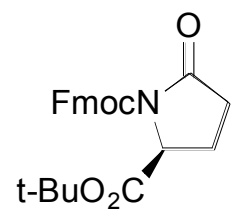

t-Butyl $N$-Fmoc-pyroglutamate (11). The general procedure for Fmoc protection of a lactam was followed using t-butyl pyroglutamate (10) $(300 \mathrm{mg}, 1.62 \mathrm{mmol})$ in THF (10 mL), LHMDS (1.539 mL, $1.539 \mathrm{mmol})$ and Fmoc-Cl (2.09 g, $8.1 \mathrm{mmol})$ in THF $(10 \mathrm{~mL})$. Flash chromatography with $20 \%$ EtOAc/petroleum ether yielded 11 as a colorless, crystalline solid $(552 \mathrm{mg}, 78 \%): \mathrm{mp} 198^{\circ} \mathrm{C}$; IR $(\mathrm{NaCl}$, $\left.\mathrm{cm}^{-1}\right) 3065,2977,2935,1798,1737,1717,1542,1450,1382,1301,1225,1156 ;{ }^{1} \mathrm{H}$ NMR $(300 \mathrm{MHz}$, $\left.\mathrm{CDCl}_{3}\right) \delta 7.75(\mathrm{~d}, 4 \mathrm{H}, 7.18 \mathrm{~Hz}), 7.41-7.29(\mathrm{~m}, 4 \mathrm{H}), 4.58-4.26(\mathrm{~m}, 4 \mathrm{H}), 2.73-2.36(\mathrm{~m}, 3 \mathrm{H}), 2.11-2.03(\mathrm{~m}$, 1H), 1.44 (s, 9H) ppm; ${ }^{13} \mathrm{C}$ NMR (75 MHz, $\left.\mathrm{CDCl}_{3}\right) \delta 172.7,170.1,151.5,143.4,143.3,141.2,127.8$, 127.2, 125.5, 125.4, 119.9, 119.8, 82.6, 69.0, 59.3, 46.6, 31.2, 27.9, 21.9 ppm; HRMS (ESI) calcd for $\mathrm{C}_{24} \mathrm{H}_{25} \mathrm{NO}_{5}(\mathrm{Na}+)$ 430.1630, found 430.1629.

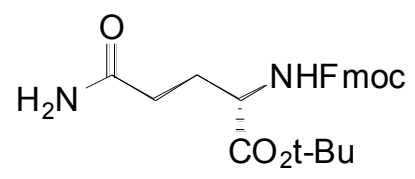

$\boldsymbol{N}$-Fmoc-(S)-glutamine t-butyl ester (12). To a solution of $\mathrm{Me}_{2} \mathrm{AlCl}(2.45 \mathrm{~mL}, 2.45 \mathrm{mmol})$ in $\mathrm{CH}_{2} \mathrm{Cl}_{2}(4 \mathrm{~mL})$ at $-8^{\circ} \mathrm{C}$ was bubbled gaseous $\mathrm{NH}_{3}$ for $4 \mathrm{~min}$. The reaction was allowed to stir at $-8^{\circ} \mathrm{C}$ for 15 minutes and allowed to warm to room temperature. Over 30 minutes $\mathrm{CH}_{2} \mathrm{Cl}_{2}$ was removed using a flow of nitrogen gas to afford a colorless slurry. The residue was added THF $(7 \mathrm{~mL})$, and let it stir at for a minute. Then t-butyl N-Fmoc-(2S)-pyroglutamate (11) (100 mg, $0.245 \mathrm{mmol})$ and $\mathrm{Eu}(\mathrm{OTf})_{3}(58.72 \mathrm{mg}$, $0.098 \mathrm{mmol})$ in THF $(1.7 \mathrm{~mL})$ was added. After 10 min reaction was quenched using $0.1 \mathrm{~N} \mathrm{HCl}(25 \mathrm{~mL})$, and EtOAc $(10 \mathrm{~mL})$. The solution was then extracted with EtOAc $(3 \times 10 \mathrm{~mL})$. Organic fractions were combined, washed with brine $(3 \times 10 \mathrm{~mL})$ and dried over $\mathrm{Na}_{2} \mathrm{SO}_{4}$. After removal of solvent under reduced 
pressure the crude product was purified by flash column chromatography with EtOAc yielded $\mathbf{1 2}$ as a colorless solid (95 mg, 91\%): mp 134-136 C; IR ( $\left.\mathrm{NaCl}, \mathrm{cm}^{-1}\right)$ 3380, 3343, 3203, 3065, 2979, 2937, 1732, 1694, 1657, 1539, 1450, 1369, 1264, 1245, 1158; ${ }^{1} \mathrm{H}$ NMR (300 MHz, $\left.\mathrm{CDCl}_{3}\right) \delta 7.75$ (d, 2H, 7.5Hz), 7.58 (d, 2H, 7.3Hz), 7.41-7.28 (m, 4H), 5.98 (br, 1H), 5.55 (d, 1H, 7.8Hz), 5.38 (b, 1H), 4.41-4.39 (m, 2H), 4.22-4.17 (m, 2H), 2.3-2.18 (m, 3H), 1.93-1.85 (m, 1H), 1.45 (s, 9H), ppm; $\left.{ }^{13} \mathrm{C} \mathrm{NMR} \mathrm{(75} \mathrm{MHz,} \mathrm{CDCl}_{3}\right) \delta$ $174.7,171.1,156.4,143.8,143.6,141.2,127.6,127,125,119.9,82.5,66.9,53.9,53.4,47.1,31.8,28.7$, 27.9 ppm; HRMS (ESI) calcd for $\mathrm{C}_{24} \mathrm{H}_{28} \mathrm{~N}_{2} \mathrm{O}_{5}(\mathrm{Na}+)$ 447.1896, found 447.1899.

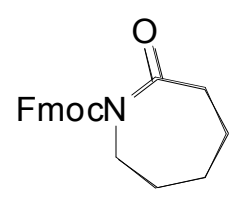

N-Fmoc-Caprolactam (16). The general procedure for Fmoc protection of a lactam was followed using ع-caprolactam (100 mg, $0.884 \mathrm{mmol})$ in THF (10 mL), LHMDS (0.8 mL, $0.8 \mathrm{mmol})$ and Fmoc-Cl $(1.14 \mathrm{~g}, 4.42 \mathrm{mmol})$ in THF (10 mL). Flash chromatography with 25\% EtOAc/petroleum ether yielded 17 as a colorless oil $(175 \mathrm{mg}, 60 \%)$ : IR $\left(\mathrm{NaCl}, \mathrm{cm}^{-1}\right)$ 3065, 3041, 3018, 2932, 2858, 1770, 1713, 1478, 1451, 1380, 1265, 1165; ${ }^{1} \mathrm{H}$ NMR (300 MHz, $\left.\mathrm{CDCl}_{3}\right) \delta 7.73$ (t, 4H, 8.1Hz), 7.41-7.31 (m, 4H), 4.49(d, 2H, $7 \mathrm{~Hz}), 4.30(\mathrm{t}, 1 \mathrm{H}, 7 \mathrm{~Hz}), 3.78(\mathrm{~m}, 2 \mathrm{H}), 2.72(\mathrm{~m}, 2 \mathrm{H}), 1.79-1.66(\mathrm{~m}, 6 \mathrm{H}) \mathrm{ppm} ;{ }^{13} \mathrm{C} \mathrm{NMR}\left(75 \mathrm{MHz}, \mathrm{CDCl}_{3}\right)$ $\delta 175.7,154.5,143.9,141.5,128,127.4,125.5,120.1,69.2,47,46.6,39.8,29.3,28.7,23.8$ ppm; HRMS (ESI) calcd for $\mathrm{C}_{21} \mathrm{H}_{21} \mathrm{NO}_{3}(\mathrm{Na}+)$ 358.1419, found 358.1420.

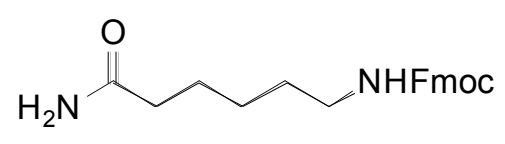

6-[(9H-fluoren-9-yl)methyloxycarbonyl $]$ aminohexanamide (17). To a solution of $\mathrm{Me}_{2} \mathrm{AlCl}$ $(180 \mu 1,0.18 \mathrm{mmol})$ in $\mathrm{CH}_{2} \mathrm{Cl}_{2}(1 \mathrm{~mL})$ at $-8^{\circ} \mathrm{C}$ was bubbled gaseous $\mathrm{NH}_{3}$ for $3.5 \mathrm{~min}$. The reaction was allowed to stir at $-8^{\circ} \mathrm{C}$ for 15 minutes and allowed to warm to room temperature. Over 30 minutes $\mathrm{CH}_{2} \mathrm{Cl}_{2}$ was removed using a flow of nitrogen gas to afford a colorless slurry. To the residue was added THF (1 $\mathrm{mL}$ ), and the solution stirred for a minute. Then $\mathrm{N}$-Fmoc-caprolactam (16) (6 mg, $0.018 \mathrm{mmol})$ and $\mathrm{Eu}(\mathrm{OTf})_{3}(4.3 \mathrm{mg}, 0.0072 \mathrm{mmol})$ in $\mathrm{THF}(1 \mathrm{~mL})$ was added. Reaction was quenched using $0.1 \mathrm{~N} \mathrm{HCl}(2$ $\mathrm{mL})$, and EtOAc $(1 \mathrm{~mL})$. The solution was then extracted with EtOAc (x3). Organic fractions were 
combined, washed with brine $(10 \mathrm{~mL})$ and dried over $\mathrm{Na}_{2} \mathrm{SO}_{4}$. After removal of solvent under reduced pressure the crude product was purified by flash column chromatography with $30 \%$ THF/EtOAc yielded

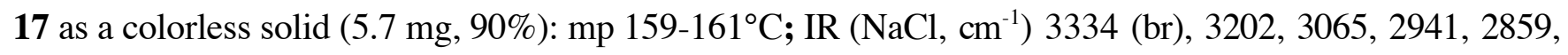
1692, 1652, 1538, 1450, 1265; ${ }^{1} \mathrm{H}$ NMR (300 MHz, $\left.\mathrm{CD}_{3} \mathrm{Cl}_{3}\right) \delta 7.75$ (d, 2H, 7Hz), 7.57 (d, 2H, 7.6Hz), 7.41-7.24 (m, 4H), $5.4(\mathrm{bs}, 2 \mathrm{H}), 4.83(\mathrm{bs}, 1 \mathrm{H}), 4.38(\mathrm{~d}, 2 \mathrm{H}, 6.4 \mathrm{~Hz}), 4.2(\mathrm{~d}, 1 \mathrm{H}, 6.5), 3.18(\mathrm{~d}, 2 \mathrm{H}, 5.9 \mathrm{~Hz})$, $2.21(\mathrm{t}, 2 \mathrm{H}, 7 \mathrm{~Hz}), 1.67-1.35(\mathrm{~m}, 6 \mathrm{H}) \mathrm{ppm} ;{ }^{13} \mathrm{C} \mathrm{NMR}\left(75 \mathrm{MHz}, \mathrm{CD}_{3} \mathrm{Cl}_{3}\right) \delta$ 156.5, 144.0, 141.3, 127.7, 127.0, 125.0, 120, 66.5, 47.3, 40.7, 35.6, 26.1, 24.9 ppm; HRMS (ESI) calcd for $\mathrm{C}_{21} \mathrm{H}_{24} \mathrm{~N}_{2} \mathrm{O}_{3}(\mathrm{Na}+)$ 375.1685 , found 375.1683 .

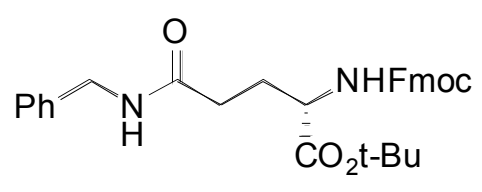

(S)-tert-butyl 2-(((9H-fluoren-9-yl)methoxy)carbonyl)-5-(benzylamino)-5-oxopentanoate (18). To a solution of $\mathrm{Me}_{2} \mathrm{AlCl}(1.23 \mathrm{~mL}, 1.23 \mathrm{mmol})$ in $\mathrm{CH}_{2} \mathrm{Cl}_{2}(2 \mathrm{~mL})$ at $-8^{\circ} \mathrm{C}$ was added benzyl amine dropwise. The reaction was allowed to stir at $-8^{\circ} \mathrm{C}$ for 15 minutes and allowed to warm to room temperature. Over 30 minutes $\mathrm{CH}_{2} \mathrm{Cl}_{2}$ was removed using a flow of nitrogen gas to afford a colorless slurry. The residue was added THF $(3.6 \mathrm{~mL})$, and let it stir at for a minute. Then t-butyl N-Fmoc-(2S)pyroglutamate (11) $(50 \mathrm{mg}, 0.123 \mathrm{mmol})$ and $\mathrm{Eu}(\mathrm{OTf})_{3}(30 \mathrm{mg}, 0.05 \mathrm{mmol})$ in THF $(0.5 \mathrm{~mL})$ was added. After 40 min reaction was quenched using $0.1 \mathrm{~N} \mathrm{HCl}(13 \mathrm{~mL})$, and EtOAc $(10 \mathrm{~mL})$. The solution was then extracted with EtOAc (x3). Organic fractions were combined, washed with brine $(10 \mathrm{~mL})$ and dried over $\mathrm{Na}_{2} \mathrm{SO}_{4}$. After removal of solvent under reduced pressure the crude product was purified by flash column chromatography with EtOAc yielded 18 as a colorless solid $(55 \mathrm{mg}, 87 \%)$ : mp 131-132 ${ }^{\circ} \mathrm{C}$, IR $\left(\mathrm{NaCl}, \mathrm{cm}^{-1}\right)$ 3304(br), 3065, 2977, 2931, 1732, 1688, 1643, 1545, 1452, 1368, 1277, 1260, 1157; ${ }^{1} \mathrm{H}$ NMR (300 MHz, $\left.\mathrm{CDCl}_{3}\right) \delta 7.73(\mathrm{~d}, 2 \mathrm{H}, 7.3 \mathrm{~Hz}), 7.59-7.55(\mathrm{~m}, 2 \mathrm{H}), 7.4-7.27(\mathrm{~m}, 9 \mathrm{H}), 6.24(\mathrm{bs}, 1 \mathrm{H}), 5.56(\mathrm{~d}, 1 \mathrm{H}, 7.3), 4.42-$ $4.36(\mathrm{~m}, 4 \mathrm{H}), 4.2-4.16(\mathrm{~m}, 2 \mathrm{H}), 2.25(\mathrm{bs}, 3 \mathrm{H}), 1.9(\mathrm{~m}, 1 \mathrm{H}), 1.45(\mathrm{~s}, \mathrm{H}) \mathrm{ppm} ;{ }^{13} \mathrm{C} \mathrm{NMR}\left(75 \mathrm{MHz}, \mathrm{CD}_{3} \mathrm{Cl}_{3}\right)$ $\delta 172.1,171.3,156.6,144.1,143.9,141.5,138.5,128.9,128,127.9,127.7,127.3,125.3,120.2,82.7,67.2$, 54.2, 47.4, 43.9, 32.8, 29.3, 28.2 ppm; HRMS (ESI) calcd for $\mathrm{C}_{31} \mathrm{H}_{34} \mathrm{~N}_{2} \mathrm{O}_{5}(\mathrm{Na}+)$ 537.2365, found 
537.2367. 